\title{
Subliminal Evaluative Conditioning Changed Implicit and Explicit Depressive Cognition
}

\author{
Akihiro Masuyama ${ }^{1, *}$, Kengo Yokomitsu ${ }^{2}$, Yuji Sakano ${ }^{3}$ \\ ${ }^{1}$ Graduate School of Comprehensive Human Sciences, University of Tsukuba, Ibaraki, Japan \\ ${ }^{2}$ Tobacco Academic Study Center, Tokyo, Japan \\ ${ }^{3}$ School of Psychological Science, Health Sciences University of Hokkaido, Hokkaido, Japan
}

\section{Email address:}

ak.masuyama@gmail.com (A. Masuyama),yokomitsuken5@gmail.com (K. Yokomitsu), sakano@hoku-iryo-u.ac.jp (Y.Sakano)

${ }^{*}$ Corresponding author

\section{To cite this article:}

Akihiro Masuyama, Kengo Yokomitsu, Yuji Sakano. Subliminal Evaluative Conditioning Changed Implicit and Explicit Depressive Cognition. American Journal of Applied Psychology. Vol. 6, No. 2, 2017, pp. 15-21. doi: 10.11648/j.ajap.20170602.11

Received: February 6, 2017; Accepted: February 18, 2017; Published: March 10, 2017

\begin{abstract}
The purpose of our study was to investigate whether subliminal evaluative conditioning could change implicit and explicit depression-related cognition. Subliminal evaluative conditioning was conducted as a form of Primed Lexical Decision Task, in which subliminally presented self-related word was followed supraminally presented positive words. For measuring implicit depression-related cognition, we used Depression Implicit Association Task (Depression IAT), and for explicit depression-related cognition, we used Depression subscale extracting from Depression And Anxiety Cognition Scale (DACSD). Furthermore, we also investigated whether the changes of implicit and explicit cognition could be last for 24 hours. As a result, we found that evaluative conditioning changed the implicit depression-related cognition. However, this changing in implicit cognition did not last for 24 hours later. In explicit depression-related cognition, evaluative conditioning by PLDT changed not all subscale but negative automatic thought for self. These results suggested that evaluative conditioning made temporally changing to implicit depression-related cognition while sustained changing to explicit depressive thought for self.
\end{abstract}

Keywords: IAT, Evaluative Conditioning, Depression, Implicit Depressive Cognition

\section{Introduction}

\subsection{Depression and Implicit Cognition}

Since "Cognitive therapy for depression" [1] was published, many research have revealed that depressive patients had negatively biased cognitive process such as automatic thoughts and thinking error [2]. These cognitive processes, usually measured by questionnaire, have been called explicit cognitive process because questionnaire measured only cognitive characteristic which participants themselves could perceive. Recently, the importance of implicit cognitive process presumed by reaction times (RTs) and error rates in cognitive tasks has been pointed [3]. Wiers, and colleagues [4] mentioned that implicit cognitive process deeply related to emerging and relapse of depression because depressed symptoms uncontrollably or non-intentionally occurred. Gotlib and Joormann [5] suggested that investigating not only explicit cognitive process but also explicit cognitive process could reveal overall cognitive process in depression.

One of the implicit cognitive process, implicit association defined as introspectively unidentified (or inaccurately identified) trace of past experience that mediates evaluation [6] has been well known. Implicit Association Test (IAT), in which binate evaluative concept (e. g. good v. s. bad) and category (e. g. flower v. s. insect) were used, could measure implicit association. Specifically, IAT mainly constructed to below 2 blocks: (1) participants were asked to classify whether presented stimulus was related to "good or flower" or "bad or insect". Then, (2) participants were asked to classify whether presented stimulus was related to "good or insect" or "bad or flower". When RTs of (1) was faster than RTs of (2), it was assumed that implicit association between "good and flower" or "bad or insect" was stronger than "good or insect" or "bad or flower". Many implicit concepts 
in various psychological research fields were revealed using by IAT. For example, there were personality aspect such as stereotype [7] and self-esteem, psychopathological aspect such as anxiety and spider phobia $[8,9]$. Also with respect to depression, several research investigated relationship between implicit self-esteem and depressed symptom. However, results in these researches were incongruent or mixed. Some research suggested that depressed symptom related to lower implicit self-esteem [10] while other reported that depressed patients had higher implicit selfesteem [11]. Because relationship between depressed symptom and implicit self-esteem was uncertain, Meites and colleagues [12] were developed depression IAT constructed to "negative-positive emotion" and "self-others", and reported that remitted depressed individuals show higher implicit depressive association than healthy controls when inducing negative mood. This result indicates the efficiency of depression IAT to detect depression-related implicit cognitive process.

\subsection{Changing Implicit Association}

Several researches revealed implicit association could change by a manipulation. Teachman and Woody [13] suggested that three-sessions of cognitive behavioral therapy improved implicit and explicit measure related to spider phobia and maintained the improvement for 2. month later follow up. Furthermore, Ozaki [14] reported that experimental manipulation changed implicit preference toward geometric figures. The experimental manipulation was simple way which participant were asked to place ovals to toward themselves whereas rectangles to away.combined with these findings, it was considered that the implicit association measured by IAT had sensitivity for manipulation including especially very simple experimental manipulation.

Evaluative conditioning, which involves changing in the negative or positive valence of stimulus that result from paring with another stimulus [15], has been well known to change evaluation and related implicit association forward specific concept or stimulus. For instance, implicit association between "pleasant" and "snack foods" changed through evaluating conditioning task in which snack food picture were paired with negatively valenced body picture. Also in the subliminal evaluative conditioning, which is modified version of evaluative conditioning in that one of paired stimuli was subliminally (usually $16 \mathrm{~ms}$ ) presented, Dijksterhuis [16] reported that pairing subliminally presented positive words with supraliminally presented self-related words increased implicit self-esteem. This result indicated that subluminal evaluative conditioning which participants only to see presented self-related words (but actually presented positive words) could change implicit cognitive process.

Despite several researches revealed that the changing implicit association through evaluative conditioning; even though there need not working awareness such as subliminal evaluative conditioning, there have been remained uncertain two points. The first point was that requires clarification is whether the effect of changing in implicit association by evaluative conditioning could last or not. Other experimental procedure was found that changing implicit association was lasting still after 24 hours [17]. To comprehend the entirely mechanism of implicit cognitive process, and the precisely effect of evaluative conditioning, it could be important to understand how long lasting the effect of changing. The second point was that requires clarification is how changing toward implicit association by subluminal evaluative conditioning affect on explicit cognition. Several theories that referred to implicit association [18] suggested that implicit association and explicit cognition was interinfluenced each other. Indeed, explicit intervention, such as education and psychotherapy, could change not only explicit cognition measured by questionnaire but also implicit cognition measured by IAT [13, 19]. Nevertheless, implicit intervention that directly manipulates implicit cognition change explicit cognition as well as implicit cognition is still remained uncertain. Considering the results about implicit depression-related cognition have been mixed in that some results suggested that implicit association between self and positive words was related to depressive symptoms [20, 21], other results suggested that implicit association between self and depressive words $[22,23]$, revealing these two points related to changes implicit association and explicit cognition could be important for fully understanding the cognitive process in depression.

Therefore, this study investigated whether subliminal evaluative conditioning could change both implicit association and explicit cognition. And if so, whether that changing could be lasting after 24 hours was also investigated. For targeting depressed-related implicit and explicit cognition, we used depression IAT to measure implicit association, subliminal evaluative conditioning task in which positive words were presented with supraliminally presented self-related words to improve depression related cognition, and questionnaire to measure explicit depressive cognition in this study. Our investigation could attribute to reveal the relationships between depression and cognition, and to perspective of Cognitive Bias Modification which directly treat the psychological symptoms by modifying cognitive bias with only using cognitive tasks [24] in that implicit association could be taken account in a therapeutic target. For this purpose, we tested follow hypotheses: 1) subliminal evaluative conditioning could change depression IAT, 2) the change of depression IAT could last until after 24 hours, and 3) explicit depressive cognitive process could be changed by subliminal evaluative conditioning in 24 hours after.

\section{Methods}

\subsection{Participants}

Thirty-six undergraduate students participated this study. They were pseudo-randomly assigned to experimental group $(\mathrm{n}=18$; female) and control group $(\mathrm{n}=18$; female). 


\subsection{Instruments}

\subsubsection{Implicit Depressive Association}

To assess implicit depressive association, we measured depression IAT [12, 25] in which used negative-positive words and self-other related words. Participants were asked to classify presented word whether left or right category. Depression IAT was constructed to seven trial blocks standardized by [26], block 1: practice for classifying selfother related words, block 2: practice for classifying positivenegative words, block 3, 4: compatible blocks of self with positive words block 5: reversed practice for block 1 , and block 6, 7: incompatible blocks of self with negative words. Each trial started with fixation of $400 \mathrm{~ms}$ duration. Next, stimulus word was presented on the computer screen until participants push left or right key. Background color was black and stimulus words were presented white $150 \mathrm{pt}$ MS gothic font. The stimulus words in depression IAT were carefully translated to Japanese so as not to spoil original nuance.

\subsubsection{Primed Lexical Decision Task (PLDT)}

To manipulate participants' cognition by subliminal evaluative conditioning, we used PLDT. Participants were asked to classify stimulus word presented on the computer screen whether written in Hiragana or Kanji. The stimulus words were neutral words (e. g. watch, note) recruited from [27], and positive words (e. g. glad, superior) recruited from self-esteem IAT [28]. Each trial was started with mask stimulus of $500 \mathrm{~ms}$ duration. Then self-related word "me" was presented $17 \mathrm{~ms}$ subliminally. After prime stimulus word, mask stimulus was again presented, and stimulus word that participants need to classify was presented. The number of trial was 40 trials in total as well as previous study [16]. Twenty trials of positive words primed self related words and twenty trials of neutral words primed nothing. Because there were no primed trials in control group, participants in control group were not conditioned.

\subsubsection{Explicit Depressive Cognition}

To assess explicit depressive cognition, we measured Depression and Anxiety Cognition Scale (DACS; [29]). DACS is a 50 items self-report questionnaire ranging from 1 to 5 , and constructed by four factors: 1) automatic thought for future, 2) that for self, 3) that for past, 4) forecasting for threaten, and 5) disgusting for situation. In this study, we used 1), 2), and 3) as DACS-D because of the three factors intimately related to depressed symptoms.

\subsection{Procedure}

As shown in figure 1, participants in each group completed DACS-D, Depression IAT as a baseline (Time 1). Then, participants in experimental group administered PLDT for subliminal evaluative conditioning whereas in control group administered LDT which all stimulus were non-primed by self-related words (Time 2). After PLDT or LDT, participants in each group administered depression IAT. And after more than 24 hours, participants came experiment room again and completed DACS-D and depression IAT. The order of depression IAT and DACS-D in Time 1. and Time 4. was well counterbalanced. This experimental procedure was approved by ethics committee of department of psychological science in Health Science University in Hokkaido.

Time1 Baseline: DACS-D, Depression IAT

Time2 Experimental group: PLDT

Control group: LDT

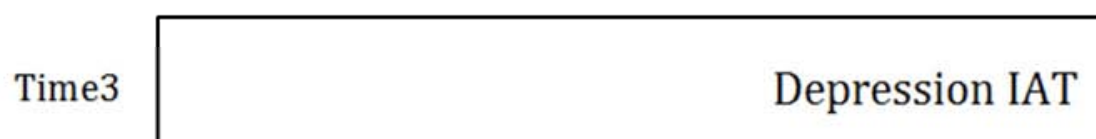

Time 4

After 24 hour

From Time 1

\section{DACS-D, Depression IAT}

Figure 1. Experimental procedure.

Note. DACS-D: Depression and Anxiety Scale depression subscale IAT: Implicit Association Test; PLDT: Primed Lexical Decision Task, LDT: Lexical Decision Task, 


\subsection{Data Analysis}

Reaction times (RT) and error trials in depression IAT were transformed to $D$ score [22] calculated by follow formulas and then used to statistical analysis.

(1) (Mean RT in block 6- mean RT in block 3)/

(SD in block 3, 6)

(2) (Mean RT in block 7- mean RT in block 4)/ (SD in block 4,7$)$

(3) $((1)+(2)) / 2$

Before $D$ score was calculated, RTs of error trials in a block were replaced to mean RT in the block adding $600 \mathrm{~ms}$.

More positive value means strong association between positive word and self-related word whereas more negative value means strong association between negative word and self-related word. Besides, RTs and error trials in PLDT and LDT were not used to statistical analysis because PLDT was only for subliminal evaluative conditioning to experimental group.

\section{Results}

\subsection{Descriptive Statistics}

As shown in Table 1, experimental and control group did not differ on sex rate $\left(x^{2}(1)=0.138, n . s.\right)$, age $(\mathrm{t}(34)=0.334$, $n$. s.), and total DACS-D score $(t(34)=1.46, n$. s.). This result suggested that there were no difference between experimental and control group in sex rate and explicit depressive cognition.

Table 1. Summary of descriptive statistics.

\begin{tabular}{lllll}
\hline & \multicolumn{2}{l}{$\begin{array}{l}\text { Experimental group } \\
(\boldsymbol{N = 1 8})\end{array}$} & $\begin{array}{l}\text { Control group } \\
(\boldsymbol{N}=\mathbf{1 8})\end{array}$ & \\
\hline & $\boldsymbol{M}$ & $\boldsymbol{S D}$ & $\boldsymbol{M}$ & $\boldsymbol{S D}$ \\
\hline Sex rate (Male: Female) & $4: 14$ & & $6: 12$ & \\
Age & 19.89 & 1.08 & 20.00 & 0.91 \\
Time 1. DACS-D & 56.50 & 24.98 & 65.33 & 27.36 \\
\hline
\end{tabular}

Note. DACS-D: Depression and Anxiety Scale depression subscale

\subsection{Implicit Cognition}

To test hypothesis 1) subliminal evaluative conditioning could change depression IAT, paired t-test was conducted for the variation of $D$ score from Time 1. to Time 3 . in each group and then revealed that the variation of $D$ score in experimental group significantly higher than that in control group $(t(34)=2.13, p<.05$; Table 2$)$. This result indicated that subliminal evaluative conditioning strengthened the implicit association between self-related words and positive words.

To test hypothesis 2) the change of depression IAT could last until after 24 hours, paired t-test was conducted for the variation of $D$ score from Time 1 . to Time 4 . in each group and revealed that the variation of $D$ score did not differ between experimental and control group $(t(34)=0.66, n$. $s$.; Table 2). This result indicated that the effect of subliminal evaluative conditioning on depressive implicit cognition did not last for 24 hours.

\subsection{Explicit Cognition}

Lastly, to test hypothesis 3) explicit depressive cognitive process could be changed by subliminal evaluative conditioning in 24 hours after, paired t-test was conducted for the variation of total DACS-D score from Time 1. to Time 4 . in each group and revealed that the variation of DACS-D score did not differ between experimental and control group $(t$ (34) $=1.36, n$. s.; Table 2). As further analysis, we conducted paired t-test for variation of each factor: automatic thought for future, for self, and for past. Paired t-test revealed that variation of automatic thought for self in experimental group significantly higher than in control group whereas variation of automatic thought for future and past did not differ between experimental and control group $(t(34)=0.45$, $n$. s.; $t(34)=0.47, n$. s., respectively; Table 2$)$. This result suggested that subliminal evaluative conditioning in which parings with self-related words and positive words were presented, improve explicit depressive cognition for self measured by questionnaire in 24 hours later.

Table 2. Amount of change in each measure.

\begin{tabular}{|c|c|c|c|c|c|c|}
\hline & & \multicolumn{2}{|c|}{ Experimental group $(N=18)$} & \multicolumn{2}{|c|}{ Control group $(N=18)$} & \multirow[t]{2}{*}{$t$} \\
\hline & & $M$ & $S D$ & $M$ & $S D$ & \\
\hline \multicolumn{7}{|l|}{$D$ score } \\
\hline & $\Delta D$ score (Time 3 -Time 1$)$ & 0.11 & 0.34 & -0.18 & 0.35 & $2.56^{*}$ \\
\hline & $\Delta D$ score (Time 4 -Time 1$)$ & 0.01 & 0.48 & -0.14 & 0.42 & $0.98 *$ \\
\hline \multicolumn{7}{|l|}{ DACS } \\
\hline & $\triangle$ DACS-D (Time 1-Time 4) & 5.67 & 9.43 & 1.78 & 7.7 & $1.36^{*}$ \\
\hline & $\Delta$ AT for past (Time 1 -Time4) & 1.22 & 3.93 & 0.67 & 3.43 & $0.45^{*}$ \\
\hline & $\Delta \mathrm{AT}$ for future (Time 1-Time4) & 1.44 & 4.12 & 0.83 & 3.59 & $0.48^{*}$ \\
\hline
\end{tabular}

Note. DACS: Depression and Anxiety Scale, DACS-D: Depression and Anxiety Scale depression subscale;

AT: Automatic Thought

\section{Discussion}

The purpose of this study was to investigate whether subliminal evaluative conditioning change implicit and explicit depressive cognition, and last the changing until after 24 hours. We found the partly effect of subliminal evaluative conditioning on implicit and explicit depressive cognition. Specifically, subliminal evaluative conditioning in which 
parings with self-related words presented subliminally and positive words presented supraliminally were used, provided temporary improvement for implicit depressive cognition and short-term (24 hours later) improvement for explicit depressive cognition.

First, the hypothesis 1) subliminal evaluative conditioning could change depression IAT, was supported. This result was congruent with that of previous studies $[16,30]$ in that evaluative conditioning changed implicit association. According to Association Propositional Evaluation (APE) model theorized by Gawronski and Bodenhausen [18], implicit cognition assumed to association network, and explicit cognition assumed to propositional information were interacted each other to form a evaluation for specific concept. In line with APE model, our result indicated that subliminal evaluative conditioning strengthened the association network between self-concept and positive words.

Second, the hypothesis 2) the change of depression IAT could last until after 24 hours was not supported. This result suggested that the effect of subliminal evaluative conditioning did not last still 24 hours later. This could be resulted in that 20 parings in subliminal evaluative conditioning were too short to maintain the changing of implicit association. Because this study is the first research that investigated the maintaining the change of implicit association by subliminal evaluative conditioning only 24 hours later, how last the effect was not clearly revealed. Further study will need to modify the measuring depression IAT shorter than 24 hours later, and the intensity or the frequency of evaluative conditioning. Specifically, checking every hour the effect of 20 parings evaluative conditioning, administering PLDT including 40. parings with positive words and self-related words in evaluative conditioning, and administering PLDT in every some hours, could detect intimately effect of subliminal evaluative conditioning.

Third, the hypothesis 3) explicit depressive cognitive process could be changed by subliminal evaluative conditioning in 24 hours after, was partly supported. That is, not all explicit depressive cognitions including automatic thought for self, past, and future but only automatic thought for self was shown improvement 24 hours later. Because participants in experimental group were conditioned paring with self-related and positive words, it was considered that only self-related depressive cognition improved. Deliberate this finding, subliminal evaluative conditioning paring with a specific concept and positive words could change the evaluation of the concept positively. Besides depression IAT, Friedman and colleagues [25] developed hopelessness IAT which could measured association between negative-positive words and time-related words (e. g. tomorrow, yesterday, future, and past). Hence, when subliminal evaluative conditioning paring with positive and future (past) was conducted, it would be possible to positively change the automatic thought for future (past).

For general discussion, our results showed subliminal evaluative conditioning provided positive changing of temporary depressive implicit association and of short-term
(24 hours later) depressive explicit cognition related to selfconcept. This gapped changings that depressive implicit association was restored at baseline whereas depressive explicit cognition for self-concept was improved from baseline in 24 hours later, could be resulted in the different characteristic of implicit and explicit cognition. Implicit association, often referred as implicit attitude, has been formed for a long time since childhood based on own experiences [31]. De Raedt [32] indicated that the implicit association had similarity to depressive schema in the context of cognitive behavior theory for depression. These characteristics of implicit association would make maintaining of experimental change to be difficult. On the one hand, explicit cognition, referred as propositional process in APE model [18], has been formed by a short term or formed with interaction of implicit association. Indeed, Gawronski \& Strack [33] reported that participants who were induced to compliance paradigm and to low situational pressure showed changing of explicit attitude but not of implicit attitude. Thus, it was considered that explicit cognition had sensitivity for changing compared to implicit association. These characteristics of explicit cognition would make lasting of change to be easy.

Contrary to our approach that urge to changing for implicit association, cognitive behavior therapy which targeted to explicit cognition in that therapist improve maladaptive cognition by a dialogue with patient, had been well known to improve psychopathological symptoms. repeatation of changing for explicit cognition in cognitive therapy was considered to decrease both negative thought for self and implicit association [34]. Teachman and colleagues [9] reported, for instance, that 12 sessions of cognitive behavior therapy for panic disorder improved not only panic and depressed symptom but also implicit association for panic. Peter \& Gawronski [35] indicated that motivation effected on implicit self-esteem mediated with explicit self-esteem. While these result suggested the top-down changing: changing for implicit association by way of changing explicit cognition, our results also suggested the bottom-up changing: changing for explicit cognition by way of changing implicit association.

As indication for therapeutic strategy, our study could show applicability to the treatment of depression. Cognitive bias modification, which directly treats the psychological symptoms by modifying cognitive bias, has been recently rapid grown treatment [36]. Because cognitive bias modification need not necessary psychiatrist and/or clinical psychologist but need only computer, cognitive bias modification has been expected to be provided to many people suffering psychological symptoms by lower cost than psychiatric and clinical psychological intervention such as pharmacotherapy and cognitive behavioral therapy. Metaanalysis showed the significance of cognitive bias modification for anxiety and depression [37]. Attention bias and memory bias have been mainly well-known target cognitive bias for treating depression and anxiety. Although we did not investigate about cognitive bias, our results 
suggested that implicit depressive association, which was measured by depression, IAT could be taken account of the target cognition for cognitive bias modification. Because our study is first research which investigated the improvement of implicit cognition using depression IAT, however, further study need more detailed explanation for implicit and explicit cognition in depression.

Lastly, we mentioned the limitation on this study. Experimental group was conditioned by paring with selfrelated and positive word but control group was not conditioned by any paring. This experimental design could be appropriated to investigate simple effect of evaluative conditioning. On this account, our results could be resulted in mere exposure effect for presenting self-related words. Further study will need setting control group to another evaluating conditioning such as paring with subliminally presented self-related words and neutral words. Because we investigated only changing for explicit and implicit depressive cognition, in addition, whether subliminal evaluative conditioning influence to depressed symptoms have been still uncertain. Of course this study has enough clinical significance in that evaluative conditioning decreased depressive cognition, further study would need to show the improvement for depressed symptoms by evaluative conditioning for the sake of suggesting new therapeutic strategy for depression.

\section{Conclusion}

In conclusion, we investigated whether subliminal evaluative conditioning improves implicit and explicit depressive cognition. Implicit cognition was measured by depression IAT, and explicit cognition was measured by questionnaire. Our results suggested that subliminal evaluative conditioning paring with self-related and positive words provided temporary changing of the implicit depressive cognition, and short-term changing of the explicit depressive cognition for self.

\section{References}

[1] Beck, A. T., Rush, A. J., Shaw, B. F., \& Emery, G. (1979). Cognitive therapy of depression. New York: Guilford Press

[2] Coyne, J., \& Gotlib, I. (1983). The role of cognition in depression: A critical appraisal. Psychological bulletin, 94, 472-505.

[3] Murrough, J. W., Iacoviello, B., Neumeister, A., Charney, D. S., \& Iosifescu, D. V. (2011). Cognitive dysfunction in depression: neurocircuitry and new therapeutic strategies. Neurobiology of Learning and Memory, 96(4), 553-63.

[4] Wiers, R. W., Teachman, B. A., \& De Houwer. (2007). Implicit cognitive processes in psychopathology: An introduction. Journal of Behavior Therapy and Experimental Psychiatry.38, 95-104.

[5] Gotlib, I. H., \& Joormann, J. (2010). Cognition and Depression: Current Status and Future Directions. Annual review of Clinical Psychology, 27, 285-312.

[6] Greenwald, A. G., Banaji, M. R. (1995). Implicit social cognition: Attitudes, self-esteem, and stereotypes. Psychological Review, 102, 4-27.

[7] Monteith, L., \& Pettit, J. W. (2011). Implicit and explicit stigmatizing attitudes and stereotypes about depression. Journal of Social and Clinical Psychology, 30, 484-505.

[8] Aikawa, A. \& Fujii, T. (2011). Using the Implicit Association Test (IAT) to measure implicit shyness. Japanese Journal of Psychology, 82, 41-48.

[9] Teachman, B. A., Marker, C. D., \& Smith-janik, S. B. (2009). Change over the course of treatment. Jounal of Conslulting and Clinical Psychology, 76, 988-1002.

[10] Franck, E., Raedt, R. D., \& Houwer, J. D. (2008). Activation of latent self-schemas as a cognitive vulnerability factor for depression: The potential role of implicit self-esteem. Cognition and Emotion, 22, 1588-1599.

[11] De Raedt, R., Schacht, R., Franck, E., \& De Hower, J. (2006). Self-esteem and depression revisited: Implicit positive selfesteem in depressed patients? Behavior Research and Therapy, 44, 1017-1028.

[12] Meites, T. M., Deveney, C. M., Steele, K. T., Holmes, A. J., \& Pizzagalli, D. A. (2008). Implicit depression and hopelessness in remitted depressed individuals. Behavior Research and Therapy, 46, 1078-1084.

[13] Teachman, B. a., \& Woody, S. R. (2003). Automatic processing in spider phobia: Implicit fear associations over the course of treatment. Journal of Abnormal Psychology, 112(1), 100-109.

[14] Ozaki, Y. (2006). Changing implicit attitudes by repeated approach or avoidance. The Japanese Journal of Experimental Social Psychology, 45, 98-110.

[15] Gast, A., De Houwer, J., \& De Schryver, M. (2012). Evaluative conditioning can be modulated by memory of the CS-US pairings at the time of testing. Learning and Motivation, 43(3), 116-126.

[16] Dijkserhuis, A. (2004). I like myself but I don't know why: Enhancing Implicit self-esteem by subliminal evaluative conditioning. Journal of Personality and Social Psychology, $86,345-355$.

[17] Kawakami, K., Dovidio, J. F., Moll, j., Hermsen, S., \& Russin, A. (2000) Just say no (to Stereotyping): Effects of training in the negation of stereotypic associations on stereotype activation. Journal of Personality and Social Psychology, 78, 871-888.

[18] Gawronski, B., \& Bodenhausen, G. V. (2006). Associative and propositional processes in evaluation: An integrative review of implicit and explicit attitude change. Psychological Bulletin, 132, 692-731.

[19] Devine, P. G., Forscher, P. S., Austin, A. J., \& Cox, W. T. L. (2012). Long-term reduction in implicit race bias: A prejudice habit-breaking intervention. Journal of Experimental Social Psychology, 48, 1267-1278.

[20] Lemmens, L. H. J. M., Roefs, A., Arntz, A., van Teeseling, H. C., Peeters, F., \& Huibers, M. J. H. (2014). The value of an implicit self-associative measure specific to core beliefs of depression. Journal of Behavior Therapy and Experimental Psychiatry, 45(1), 196-202. 
[21] De Raedt, R., Schacht, R., Franck, E., \& De Houwer, J. (2006). Self-esteem and depression revisited: implicit positive self-esteem in depressed patients? Behaviour Research and Therapy, 44(7), 1017-28.

[22] Grano, C., Muzi, M., Pompili, M., Erbuto, E., \& Violani, C. (2016). Measuring the automatic negative self-schema: New evidence for the construct and criterion validity of the Depression Implicit Association. Self and Identity, 15(5), 599613.

[23] Creemers, D. H. M., Scholte, R. H. J., Engels, R. C. M. E., Pieters, S., \& Wiers, R. W. (2013). Acute stress increases implicit depression and decreases implicit self-esteem. Journal of Experimental Psychopathology, 4, 118-132.

[24] Rinck, M. (2016). CBM research needs more power: Commentary on the special issue on cognitive bias modification. Journal of Behavior Therapy and Experimental Psychiatry.

[25] Friedman, M. A., Nosek, B. A., Miller, I. W., Gordon, K. C., \& Banaji, M. R. (2001). Implicit hopelessness and severity of depressive symptoms. Unpublished manuscript.

[26] Greenwald, A. G., Nosek, A. B., \& Banaji, M. R. (2003). Understanding and Using the Implicit Association Test: I. An Improved Scoring Algorithm. Journal of Personality and Social Psychology, 85, 197-216.

[27] Fuiji, T., Ikeda, R., Uebuchi, H. (2009). The priming effect on the achievement motivation. Bulletin of Tokyo Gakugei University. Educational sciences, 60, 131-139.

[28] Greenwald, A. G., \& Farnham, S. D. (2000). Using the Implicit Association Test to measure self-esteem and selfconcept. Journal of Personality and Social Psychology, 79, 1022-1038.

[29] Fukui, I. (1998). Development of depression and anxiety cognition scale: toward the construction of a cognitive- behavioral model of depression and anxiety. Japanese journal of behavior therapy, 24, 57-70.

[30] Grumm, M., Nestler, S., \& Collani, G. Von. (2009). Changing explicit and implicit attitudes: The case of self-esteem. Journal of Experimental Social Psychology, 45, 327-335.

[31] Roefs, A., Hujiding, J., Smulders, F. T. Y., MacLeod, C. M., Jong, P, J., Wiers, R. W., \& Jansen, A. T. M. (2011). Implicit measures of association in psychopathology research. Psychological Bulletin, 137, 149-193.

[32] De Raedt, R. (2006). Activation of implicit self-schemas and a difficulty to disengage from negative cognitions in depression: An experimental psychopathology approach. Psychological Belgica, 46, 117-130.

[33] Gawronski, B., \& Strack, F. (2004). On the propositional nature of cognitive consistency:Dissonance changes explicit, but not implicit attitudes. Journal of Experimental Social Psychology, 40, 535-542.

[34] Teasdale, J. D., Moore, R. G., Hayhurst, H., Pope, M., Williams, S., \& Segal, Z. V. (2002). Metacognitive awareness and prevention of relapse in depression: Empirical evidence. Journal of Consulting and Clinical Psychology, 70, 275-287.

[35] Peters, K. R., \& Gawronski, B. (2011). Mutual influences between the implicit and explicit self-concepts: The role of memory activation and motivated reasoning. Journal of Experimental Social Psychology, 47(2), 436-442.

[36] Hertel, P. T., \& Mathews, A. (2011). Cognitive Bias Modification: Past Perspectives, Current Findings, and Future Applications. Perspectives on Psychological Science, 6, 521536.

[37] Hallion, L. S., \& Ruscio, A. M. (2011). A meta-analysis of the effect of cognitive bias modification on anxiety and depression. Psychological Bulletin, 137(6), 940-958. 